ELECTRONIC RESEARCH ANNOUNCEMENTS OF THE AMERICAN MATHEMATICAL SOCIETY

Volume 5, Pages 146-156 (December 9, 1999)

S $1079-6762(99) 00073-6$

\title{
STATE-SUM INVARIANTS OF KNOTTED CURVES AND SURFACES FROM QUANDLE COHOMOLOGY
}

\author{
J. SCOTT CARTER, DANIEL JELSOVSKY, SEIICHI KAMADA, LAUREL LANGFORD, \\ AND MASAHICO SAITO \\ (Communicated by Walter Neumann)
}

\begin{abstract}
State-sum invariants for classical knots and knotted surfaces in 4-space are developed via the cohomology theory of quandles. Cohomology groups of quandles are computed to evaluate the invariants. Some twist spun torus knots are shown to be noninvertible using the invariants.
\end{abstract}

\section{INTRODUCTION}

A cohomology theory for racks (self-distributive groupoids, defined below) was defined and the general framework for defining invariants of codimension 2 embeddings was outlined in [12 and 13] from an algebro-topological viewpoint. The present paper announces state-sum invariants, defined diagrammatically using knot diagrams and quandle cocycles, for both classical knots in 3-space and knotted surfaces in 4-space. The invariant is used to give a proof that some 2-twist spun torus knots are noninvertible (not equivalent to the same knot with its orientation reversed). Details of proofs and computations can be found in [4] and [5].

This cocycle invariant can be seen as an analog of the Dijkgraaf-Witten invariants for 3-manifolds [10] in that colorings and cocycles are used to define state-sum invariants. Our inspiration for the definition of these invariants is found in Neuchl's paper [27] where related cocycles (in quantum doubles of finite groups) are used to show representations of a Hopf category form a braided monoidal 2-category. Our definition was derived from an attempt to construct a 2-functor from the braided 2-category of knotted surfaces as summarized in [1] and presented in detail in [2], to another 2-category constructed from quandles.

The noninvertibility for certain classical knots had been presumed since the 1920's but proved first by Trotter 29], and subsequently by Kawauchi 24] and Hartley [17] (see also [23]). Fox [14] presented a noninvertible knotted sphere using Alexander modules, which, however, fail to detect the noninvertibility of the 2-twist spin trefoil. D. Ruberman informed us that Levine pairings and Casson-Gordon invariants detect noninvertibility of some twist spun knots [18, 28]. Thus relations

Received by the editors May 28, 1999.

1991 Mathematics Subject Classification. Primary 57M25, 57Q45; Secondary 55N99, 18G99.

Key words and phrases. Knots, knotted surfaces, quandle cohomology, state-sum invariants.

The third author was supported by a Fellowship from the Japan Society for the Promotion of Science.

(C)1999 American Mathematical Society 
between these invariants and the state-sum invariants deserve investigation. Furthermore, since state-sums can be used to define Jones polynomials [15] and their generalizations, and quandles encode fundamental group information, relations of the invariants defined herein to both quantum and classical knot invariants are expected.

The paper is organized as follows. The cohomology theory of quandles is defined in Section 2. In Section 3 the invariants are defined. Summaries of computations of quandle cocycles for some quandles and evaluations of the invariants are presented in Section 4 Properties of the invariant and applications are presented in Section 5

\section{QUANDLES, RACKS, AND THEIR COHOMOLOGY}

Definition 2.1. A quandle, $X$, is a set with a binary operation $(a, b) \mapsto a * b$ such that

(I) For any $a \in X, a * a=a$.

(II) For any $a, b \in X$, there is a unique $c \in X$ such that $a=c * b$.

(III) For any $a, b, c \in X$, we have $(a * b) * c=(a * c) *(b * c)$.

A rack is a set with a binary operation that satisfies (II) and (III).

A typical example of a quandle is a group $X=G$ with $n$-fold conjugation as the quandle operation: $a * b=b^{-n} a b^{n}$. Racks and quandles have been studied in [3, 11, 16] 22 and 25, for example. The axioms for a quandle correspond respectively to the Reidemeister moves of type I, II, and III (see also [11, 221). Indeed, knot diagrams were one of the motivations to define such an algebraic structure.

Definition 2.2. Let $X$ be a rack, and let $A$ be an abelian group, written additively. The cochain group $C^{n}=C^{n}(X ; A)$ is the abelian group of functions $f: \mathrm{FA}\left(X^{n}\right) \rightarrow$ $A$ from the free abelian group generated by $n$-tuples of elements of $X$ to the abelian group $A$. The coboundary homomorphism $\delta: C^{n} \rightarrow C^{n+1}$ is defined by

$$
\begin{aligned}
(\delta f)\left(x_{0}, \ldots, x_{n}\right)= & \sum_{i=1}^{n}(-1)^{i-1} f\left(x_{0}, \ldots, \hat{x}_{i}, \ldots, x_{n}\right) \\
& +\sum_{j=1}^{n}(-1)^{j} f\left(x_{0} * x_{j}, \ldots, x_{j-1} * x_{j}, x_{j+1}, \ldots, x_{n}\right) .
\end{aligned}
$$

(Note: Neither sum includes a 0th term as these terms cancel.)

A routine calculation shows that

Lemma 2.3. The cochain group and the boundary homomorphism form a cochain complex.

Definition 2.4. The cohomology groups of the above complex are called the rack cohomology groups and are denoted by $H_{\text {rack }}^{n}(X, A)$. Also, the groups of cocycles and coboundaries are denoted by $Z_{\text {rack }}^{n}(X, A)$ and $B_{\text {rack }}^{n}(X, A)$ respectively. Their elements are called $n$-cocycles and $n$-coboundaries, respectively. This definition coincides with the cohomology theory defined in [12] and [13].

For applications, we are interested in the case when $X$ is a quandle, so we will intersect the cocycles and coboundaries with a subset that captures axiom (I) and its consequences in higher dimensions. Thus we modify the above definition as follows. Let $P^{n}=\left\{f \in C^{n}: f(\vec{x})=0\right.$ for all $\vec{x}$ such that $x_{j}=x_{j+1}$ for some $\left.j\right\}$. 


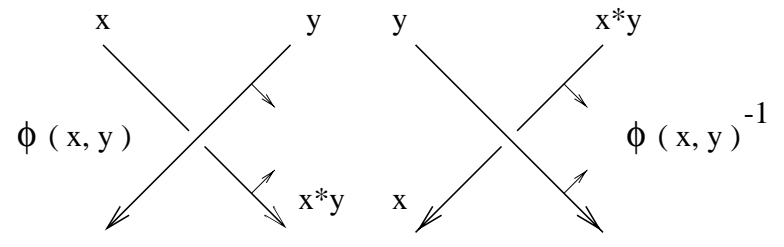

FIGURE 1. Coloring condition and weights for crossings.

Let $Z^{n}=Z_{\text {rack }}^{n} \cap P^{n}$, and $B^{n}=B_{\text {rack }}^{n} \cap P^{n}$. A straightforward calculation gives: if $f \in P^{n}$, then $\delta f \in P^{n+1}$ if $X$ is a quandle.

Definition 2.5. Define

$$
H_{Q}^{n}(X, A)=H^{n}(X, A)=\left(P^{n} \cap Z_{\text {rack }}^{n}\right) /\left(\delta P^{n-1}\right) .
$$

This group is called the quandle cohomology group. The elements $f \in Z^{n}(X, A)$ are called quandle $n$-cocycles or simply $n$-cocycles.

Throughout this paper we assume that our quandles are finite and an $n$-cocycle means a quandle $n$-cocycle.

\section{Cocycle invariants OF KNOTtings}

\subsection{Cocycle invariants of classical knots.}

Definition 3.1. A color (or coloring) on an oriented classical knot diagram is a function $\mathcal{C}: R \rightarrow X$, where $X$ is a fixed quandle and $R$ is the set of over-arcs in the diagram, satisfying the condition depicted in Figure1, In the figure, a crossing with over-arc, $r$, has color $\mathcal{C}(r)=y \in X$. The under-arcs $r_{1}$ and $r_{2}$ are colored $\mathcal{C}\left(r_{1}\right)=x$ and $\mathcal{C}\left(r_{2}\right)=x * y$, where $r_{1}$ is the arc away from which the co-orientation arrow of the over-arc points. Note that locally the colors do not depend on the orientation of the under-arc. If the pair of the co-orientation of the over-arc and that of the under-arc matches the (right-hand) orientation of the plane, then the crossing is called positive; otherwise it is negative.

Definition 3.2. Let $X$ be a finite quandle. Pick a quandle 2-cocycle $\phi \in Z^{2}(X, A)$, and write the coefficient group, $A$, multiplicatively. Consider a crossing in the diagram. For each coloring of the diagram, evaluate the 2-cocycle on two of the three quandle colors that appear near the crossing. One such color is the color on the over-arc and is the second argument of the 2-cocycle. The other color should be chosen to be the color on the under-arc away from which the normal arrow points; this is the first argument of the cocycle.

In Figure 1 the two possible oriented and co-oriented crossings are depicted. The left is a positive crossing, and the right is negative. Let $\tau$ denote a crossing, and $\mathcal{C}$ denote a coloring. When the colors of the segments are as indicated, the (Boltzmann) weights of the crossing, $B(\tau, \mathcal{C})=\phi(x, y)^{\epsilon(\tau)}$, are as shown. These weights are assignments of cocycle values to the colored crossings where the arguments are as defined in the previous paragraph.

The partition function, or a state-sum, is the expression

$$
\sum_{\mathcal{C}} \prod_{\tau} B(\tau, \mathcal{C})
$$




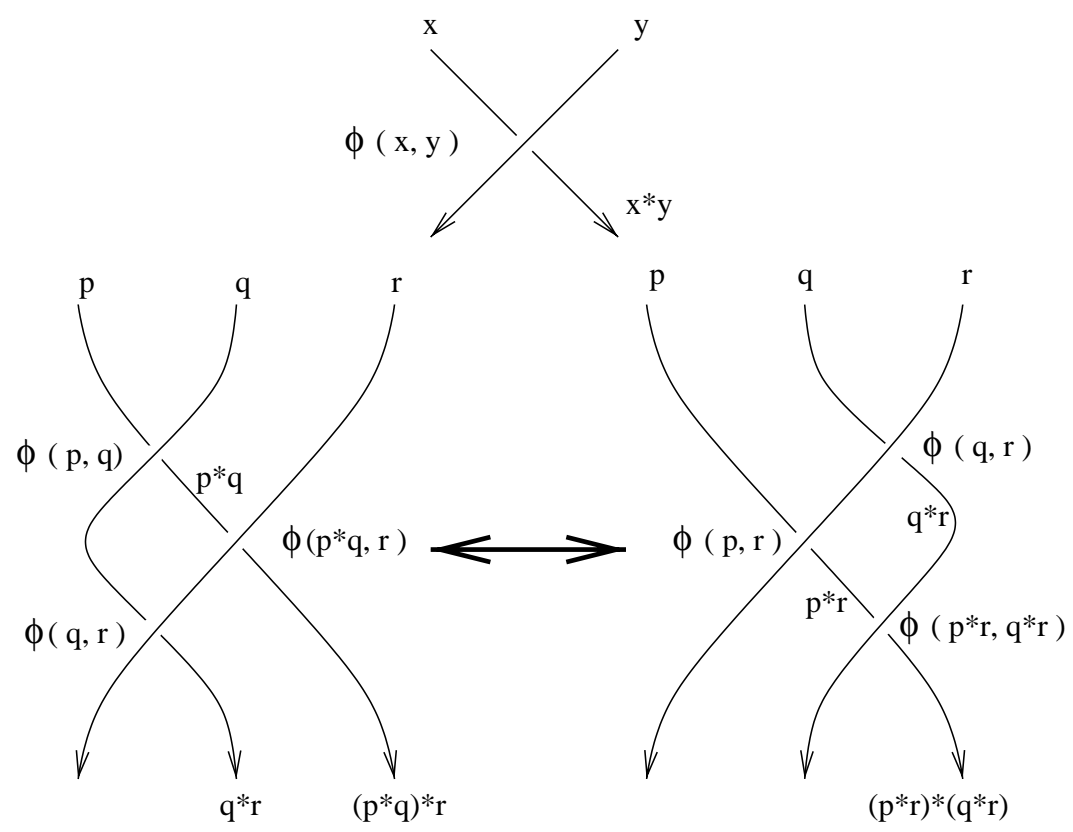

Figure 2. The 2-cocycle condition and the Reidemeister type III move.

The product is taken over all crossings of the given diagram, and the sum is taken over all possible colorings. The values of the partition function are taken to be in the group ring $\mathbf{Z}[A]$, where $A$ is the coefficient group.

Reidemeister moves are checked to prove

Theorem 3.3. The partition function is invariant under Reidemeister moves, so that it defines an invariant of knots and links. Thus it will be denoted by $\Phi(K)$ (or $\Phi_{\phi}(K)$ to specify the 2 -cocycle $\phi$ used).

Proposition 3.4. If $\Phi_{\phi}$ and $\Phi_{\phi^{\prime}}$ denote the state-sum invariants defined from cohomologous cocycles $\phi$ and $\phi^{\prime}$, then $\Phi_{\phi}=\Phi_{\phi^{\prime}}$ (so that $\Phi_{\phi}(K)=\Phi_{\phi^{\prime}}(K)$ for any link $K)$. In particular, the state-sum is equal to the number of colorings of a given knot diagram if the 2-cocycle used for the Boltzmann weight is a coboundary.

3.2. Cocycle invariants for knotted surfaces. First we recall the notion of knotted surface diagrams. See [7] for details and examples. Let $f: F \rightarrow \mathbf{R}^{4}$ denote a smooth embedding of a closed surface $F$ into 4 -dimensional space. By deforming the map $f$ slightly by an ambient isotopy of $\mathbf{R}^{4}$ if necessary, we may assume that $p \circ f$ is a general position map, where $p: \mathbf{R}^{4} \rightarrow \mathbf{R}^{3}$ denotes the orthogonal projection onto an affine subspace.

Along the double curves, one of the sheets (called the over-sheet) lies farther than the other (under-sheet) with respect to the projection direction. The undersheets are coherently broken in the projection, and such broken surfaces are called knotted surface diagrams.

When the surface is oriented, we take normal vectors $\vec{n}$ to the projection of the surface such that the triple $\left(\vec{v}_{1}, \vec{v}_{2}, \vec{n}\right)$ matches the orientaion of 3 -space, where 
$\left(\vec{v}_{1}, \vec{v}_{2}\right)$ defines the orientation of the surface. Such normal vectors are defined on the projection at all points other than the isolated branch points.

Definition 3.5. A color on an oriented (broken) knotted surface diagram is a function $\mathcal{C}: R \rightarrow X$, where $X$ is a fixed quandle and where $R$ is the set of regions in the broken surface diagram, satisfying the following condition at the double point set.

At a double point curve, two coordinate planes intersect locally. One is the over-sheet $r$, the other is the under-sheet, and the under-sheet is broken into two components, say $r_{1}$ and $r_{2}$. A normal of the over-sheet $r$ points to one of the components, say $r_{2}$. If $\mathcal{C}\left(r_{1}\right)=x \in X, \mathcal{C}(r)=y$, then we require that $\mathcal{C}\left(r_{2}\right)=x * y$.

It is shown that the above colorings are consistent near each triple point.

Definition 3.6. Note that when three sheets form a triple point, they have relative positions top, middle, bottom with respect to the projection direction of $p: \mathbf{R}^{4} \rightarrow$ $\mathbf{R}^{3}$. The sign of a triple point is positive if the normals of top, middle, bottom sheets in this order match the right-handed orientation of the 3-space. Otherwise the sign is negative.

Definition 3.7. A (Boltzmann) weight at a triple point, $\tau$, is defined as follows. Let $R$ be the octant from which all normal vectors of the three sheets point outwards; let a coloring $\mathcal{C}$ be given. Let $p, q, r$ be colors of the bottom, middle, and top sheets respectively, that bound the region $R$. Let $\epsilon(\tau)$ be the sign of the triple point, and $\theta$ be a quandle 3 -cocycle. Then the Boltzmann weight $B(\tau, \mathcal{C})$ assigned to $\tau$ with respect to $\mathcal{C}$ is defined to be $\theta(p, q, r)^{\epsilon(\tau)}$, where $p, q, r$ are colors described above.

Definition 3.8. The partition function, or a state-sum, is the expression

$$
\sum_{\mathcal{C}} \prod_{\tau} B(\tau, \mathcal{C}),
$$

where $B(\tau, \mathcal{C})$ is the Boltzmann weight assigned to $\tau$. As in the classical case, the value is taken to be in the group ring $\mathbf{Z}[A]$, where $A$ is the coefficient group written multiplicatively.

Roseman [30] generalized Reidemeister moves to dimension 4. By showing that the invariant remains unchanged under Roseman moves, we have

Theorem 3.9. The partition function does not depend on the choice of knotted surface diagram. Thus it is an invariant of knotted surfaces $F$, and denoted by $\Phi(F)$ (or $\Phi_{\theta}(F)$ to specify the 3 -cocycle $\theta$ used).

Proposition 3.10. If $\Phi_{\theta}$ and $\Phi_{\theta^{\prime}}$ denote the state-sum invariants defined from cohomologous cocycles $\theta$ and $\theta^{\prime}$, then $\Phi_{\theta}=\Phi_{\theta^{\prime}}$ (so that $\Phi_{\theta}(K)=\Phi_{\theta^{\prime}}(K)$ for any knotted surface $K)$. In particular, if $\theta$ is a 3-coboundary, then the state-sum defined above is equal to the number of colorings.

\section{Cocycles of Quandles and evaluations of invariants}

4.1. Computing cohomology. Suppose that the coefficient group $A$ is either a cyclic group, $\mathbf{Z}, \mathbf{Z}_{n}$, or the rational numbers, $\mathbf{Q}$. Define a characteristic function

$$
\chi_{x}(y)= \begin{cases}1 & \text { if } x=y, \\ 0 & \text { if } x \neq y,\end{cases}
$$


from the free abelian group $\mathrm{FA}\left(X^{n}\right)$ to the group $A$. The set $\left\{\chi_{x}: x \in X^{n}\right\}$ of such functions spans the group $C_{\text {rack }}^{n}(X, A)$ of cochains. Thus if $f \in C_{\text {rack }}^{n}(X, A)$ is a cochain, then for some integers $C_{x}$,

$$
f=\sum_{x \in X^{n}} C_{x} \chi_{x}
$$

We are interested in those $f$ 's in $P^{n} ;$ i.e. those homomorphisms that vanish on $S=\left\{\left(x_{1}, \ldots, x_{n}\right) \in X^{n}: x_{j}=x_{j+1}\right.$ for some $\left.j\right\}$. So we can write

$$
f=\sum_{x \in X^{n} \backslash S} C_{x} \chi_{x} .
$$

We used these characteristic functions to compute cohomology groups. We turn now to examples.

Definition 4.1 (11]). A rack is called trivial if $x * y=x$ for any $x, y$.

The dihedral quandle $R_{n}$ of order $n$ is the quandle consisting of reflections of the regular $n$-gon with the conjugation as operation. The dihedral group $D_{2 n}$ has a presentation

$$
\left\langle x, y \mid x^{2}=1=y^{n}, x y x=y^{-1}\right\rangle,
$$

where $x$ is a reflection and $y$ is a rotation of a regular $n$-gon. The set of reflections $R_{n}$ in this presentation is $\left\{a_{i}=x y^{i}: i=0, \ldots, n-1\right\}$, where we use the subscripts from $\mathbf{Z}_{n}$ in the following computations. The operation is

$$
a_{i} * a_{j}=a_{j}^{-1} a_{i} a_{j}=x y^{j} x y^{i} x y^{j}=x y^{j} y^{-i} y^{j}=a_{2 j-i} .
$$

Hence $R_{n}=\mathbf{Z}_{n}$ as a set, with quandle operation $i * j=2 j-i(\bmod n)$. Compare with the well known $n$-coloring of knot diagrams [14].

Let $\Lambda=\mathbf{Z}\left[T, T^{-1}\right]$ be the Laurent polynomial ring over the integers. Then any $\Lambda$-module $M$ has a quandle structure defined by $a * b=T a+(1-T) b$ for $a, b \in M$. For any Laurent polynomial $h(T), \mathbf{Z}_{n}\left[T, T^{-1}\right] /(h(T))$ is a quandle. We call such quandles $(\bmod n)$-Alexander quandles. We denote the 4-element quandle $\mathbf{Z}_{2}\left[T, T^{-1}\right] /\left(T^{2}+T+1\right)$ by $S_{4}$.

We used Maple and Mathematica to compute some of the following results. More computations can be found in [5].

- $H_{Q}^{2}\left(R_{4}, \mathbf{Z}\right) \cong \mathbf{Z} \times \mathbf{Z}$.

- $H_{Q}^{2}\left(R_{6}, \mathbf{Z}_{q}\right) \cong \mathbf{Z}_{q} \times \mathbf{Z}_{q}$ for prime numbers $q$.

- $H_{Q}^{2}\left(\mathbf{Z}_{8}\left[T, T^{-1}\right] /(T-5), \mathbf{Z}_{q}\right) \cong\left(\mathbf{Z}_{q}\right)^{n}$ where $n=16$ for $q=2$ and $n=12$ for all other primes $2<q<20$.

- $H_{Q}^{2}\left(S_{4} ; \mathbf{Z}_{2}\right) \cong \mathbf{Z}_{2}, H_{Q}^{2}\left(S_{4} ; A\right)=0$ for any other $A$ without elements of order 2.

- $H_{Q}^{2}\left(\mathbf{Z}_{3}\left[T, T^{-1}\right] /\left(T^{2}+1\right), \mathbf{Z}_{3}\right) \cong \mathbf{Z}_{3}$ and is 0 for all primes $q$ except $3,1<q<$ 20 .

- $H_{Q}^{3}\left(R_{3}, \mathbf{Z}_{3}\right) \cong \mathbf{Z}_{3}, H_{Q}^{3}\left(R_{3}, \mathbf{Z}\right) \cong 0$.

- $H_{Q}^{3}\left(R_{4}, \mathbf{Z}_{q}\right) \cong\left(\mathbf{Z}_{q}\right)^{n}$ where $n=8$ for $q=2$ and $n=2$ for all other primes $q$.

- $H_{Q}^{3}\left(R_{6}, \mathbf{Z}_{q}\right) \cong\left(\mathbf{Z}_{q}\right)^{n}$ where $n=4$ for $q=3$ and $n=2$ for all other primes $2<q<12$.

- $H_{Q}^{3}\left(S_{4}, \mathbf{Z}_{2}\right) \cong\left(\mathbf{Z}_{2}\right)^{3}, H_{Q}^{3}\left(S_{4}, \mathbf{Z}_{4}\right) \cong\left(\mathbf{Z}_{2}\right)^{2} \times \mathbf{Z}_{4}$.

- $H_{Q}^{3}\left(\mathbf{Z}_{2}\left[T, T^{-1}\right] /\left(T^{2}+1\right), \mathbf{Z}_{q}\right) \cong\left(\mathbf{Z}_{q}\right)^{n}$ where $n=8$ for $q=2$ and $n=2$ for all other primes $2<q<12$. 


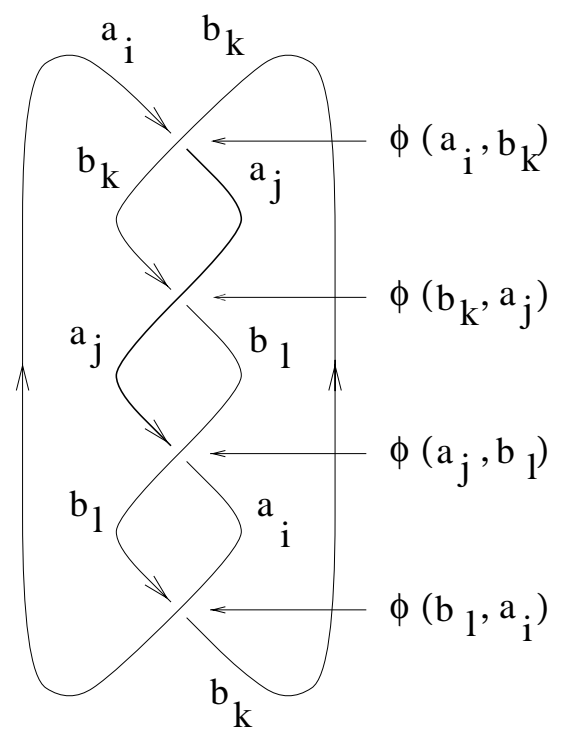

Figure 3. Computations for $(4,2)$-torus link.

\subsection{Evaluating the invariants.}

Example $4.2((4,2)$-torus link). We compute the state-sum invariant for the $(4,2)$ torus link with $X=R_{4}$. See Figure 3 .

Denote the generator of $\mathbf{Z}$ by $t$ with multiplicative notation $\left(\mathbf{Z}=\left\{t^{n}\right\}\right)$. The invariant takes values in $\mathbf{Z}\left[t, t^{-1}\right]$. Pick a noncoboundary cocycle, for example, $\phi=\chi_{\left(a_{1}, b_{1}\right)} \chi_{\left(a_{1}, b_{2}\right)}$ (in multiplicative notation). Here we use the notation $a_{1}=0$, $b_{1}=1, a_{2}=2, b_{2}=3$. Geometrically $a_{1}, a_{2}, b_{1}$, and $b_{2}$ represent the reflections of a square about the horizontal axis, vertical axis, the line $y=x$, the line $y=-x$, respectively.

In Figure 3] a specific color and the corresponding weights are shown. All possible colors are obtained as follows. If only one quandle element is used, the color's state-sum contribution is trivial (1). Since $R_{4}$ has 4 elements, there are 4 such possibilities. If one component is colored by $a_{1}$, and the other by $a_{2}$, there are no crossings of weight $\phi\left(a_{1}, b_{j}\right)^{ \pm 1}$ for $j \in\{1,2\}$, so these two colorings give trivial state-sum contributions as well. Coloring one component by $b_{1}$ and the other by $b_{2}$ produces 2 similar cases. When one component is colored by the $a$ 's and the other by the $b$ 's, the color contributes $t$ to the state-sum. There are 8 such colors, one of which is depicted in Figure 3. Since these cases cover all possible colors, the state-sum is $8+8 t=8(1+t)$.

Example 4.3. For $S_{4}=\mathbf{Z}_{2}\left[T, T^{-1}\right] /\left(T^{2}+T+1\right)$ with the coefficient $A=\mathbf{Z}_{2}$, we used the cocycle $\phi=\prod \chi_{(a, b)}$ where the product is taken over all pairs $(a, b)$ such that $a, b \in\{0,1, T+1\}$ and $a \neq b$ (the element $T$ is excluded). The invariants take the following values for tabulated knots through nine crossings.

- $4(1+3 t)$ for $3_{1}, 4_{1}, 7_{2}, 7_{3}, 8_{1}, 8_{4}, 8_{11}, 8_{13}, 9_{1}, 9_{6}, 9_{12}, 9_{13}, 9_{14}, 9_{21}, 9_{23}, 9_{35}$, $9_{37}$.

- $16(1+3 t)$ for $8_{18}, 9_{40}$. 
- 16 for $8_{5}, 8_{10}, 8_{15}, 8_{19}-8_{21}, 9_{16}, 9_{22}, 9_{24}, 9_{25}, 9_{28}-9_{30}, 9_{36}, 9_{38}, 9_{39}, 9_{41}-9_{45}$, 949 .

- 4 otherwise.

For a certain cocycle of $\mathbf{Z}_{3}\left[T, T^{-1}\right] /\left(T^{2}+1\right)$ with the coefficient $A=\mathbf{Z}_{3}$, we get - $9\left(1+4 t+4 t^{2}\right)$ for $4_{1}, 5_{2}, 8_{3}, 8_{17}, 8_{18}, 8_{21}, 9_{6}, 9_{7}, 9_{11}, 9_{24}, 9_{26}, 9_{37}-9_{39}, 9_{47}$. - $297+216 t+216 t^{2}$ for $9_{40}$.

- 81 for $6_{3}, 8_{2}, 8_{19}, 8_{24}, 9_{12}, 9_{13}, 9_{46}$.

- 9 otherwise.

Example 4.4. Zeeman's [35] twist spun knots and links form an important class of knotted surfaces in 4 -space. Let $\tau^{k} T(n, m)$ denote the $k$-twist spun torus knot of type $(n, m)$. For certain cocycles in the indicated groups, we have

- $\Phi\left(\tau^{2} T(2,3)\right)=3+6 t$, with a cocycle in $Z^{3}\left(R_{3}, \mathbf{Z}_{3}\right)$.

- $\Phi\left(\tau^{2} T(2,4)\right)=12+4 t$, with a cocycle in $Z^{3}\left(R_{4}, \mathbf{Z}_{2}\right)$.

- $\Phi\left(\tau^{2} T(2,5)\right)=5+10 t+10 t^{4}$, with a cocycle in $Z^{3}\left(R_{5}, \mathbf{Z}_{5}\right)$.

\section{TOPOLOGICAL PROPERTIES AND APPLiCATIONS}

5.1. Relations to linking numbers. For some quandles, the invariant is related to the linking number. The trivial quandle $T_{n}$ of $n$ elements is an example.

Theorem 5.1. For any cocycle of $T_{n}$, where $n$ is any positive integer, and for any link $L$, the state-sum $\Phi(L)$ is a function of pairwise linking numbers.

It is shown that any cocycle of $R_{4}$ which is not a coboundary can be written as $\phi=\lambda_{1}^{u} \lambda_{2}^{v} \in Z^{2}\left(R_{4}, \mathbf{Z}\right)$ for some integers $u$ and $v$, where $\lambda_{1}=\chi_{\left(a_{1}, b_{1}\right)} \chi_{\left(a_{1}, b_{2}\right)}$ and $\lambda_{2}=\chi_{\left(a_{2}, b_{1}\right)} \chi_{\left(a_{2}, b_{2}\right)}$.

Theorem 5.2. The state-sum invariant $\Phi(L)$ with respect to the cocycle $\phi=\lambda_{1}^{u} \lambda_{2}^{v}$ of $R_{4}$, of any $n$-component link $L=K_{1} \cup \cdots \cup K_{n}$ such that any pairwise linking number is even, is of the form

$$
\Phi(L)=2^{n}\left(\sum_{A \in \mathcal{P}(\mathcal{K})} t^{(u+v) \ell k(A, B) / 2}\right),
$$

where $\mathcal{K}=\left\{K_{1}, \ldots, K_{n}\right\}, \mathcal{P}(\mathcal{K})$ denotes its power set, $B=\mathcal{K} \backslash A$.

For example, for 1-, 2-, 3-component links L, respectively,

$$
\begin{aligned}
& \Phi(L)=4 \\
& \Phi(L)=8\left(1+t^{(u+v) \ell k(L) / 2}\right) . \\
& \Phi(L)=16\left(1+\sum_{i, j=1, i<j}^{3} t^{(u+v)\left(\ell k(L)-x_{i, j}\right) / 2}\right) .
\end{aligned}
$$

By finding links with appropriate colors, one can derive algebraic consequences. Geometric proofs of algebraic facts are characteristic to quantum topology.

Theorem 5.3. For any positive integers $n, m>1$, there exists a link $L$ whose cocycle invariant is nontrivial with the Alexander quandle $\mathbf{Z}_{n}\left[T, T^{-1}\right] /\left(T^{2 m}-1\right)$.

In particular, $H^{2}\left(\mathbf{Z}_{n}\left[T, T^{-1}\right] /\left(T^{2 m}-1\right), \mathbf{Z}\right) \neq 0$ for any $n, m>1$. 
Recall that the linking number of a 2-component classical link $L=K_{1} \cup K_{2}$ can be defined by counting the crossing number with signs $( \pm 1)$ where the component $K_{1}$ crosses over $K_{2}$ (33], see also the preceding section). This definition is generalized to linked surfaces. Let $F=K_{1} \cup \cdots \cup K_{n}$ be a linked surface, where $K_{i}, i=1, \ldots, n$, are components.

Definition 5.4. Let $T_{ \pm}(i, j, k)$ denote the number of positive and negative, respectively, triple points such that the top, middle, and bottom sheets are from components $K_{i}, K_{j}$, and $K_{k}$ respectively. Such a triple point is called of type $(i, j, k)$. Then define $T(i, j, k)=T_{+}(i, j, k)-T_{-}(i, j, k)$.

Theorem 5.5. The numbers $T(i, j, k)$ are invariants of isotopy classes of $F$ if $i \neq j$ and $j \neq k$.

Using the above triple linking numbers, nontriviality of the invariant with $T_{n}$ is obtained.

Theorem 5.6. Let $X=\{x, y, z\}$ be the trivial quandle of three elements and $\theta \in$ $Z^{3}(X, \mathbf{Z})$ be the cocycle $\chi_{(x, y, z)}$ which is the characteristic function:

$$
\chi_{(x, y, z)}(a, b, c)= \begin{cases}t & \text { if }(a, b, c)=(x, y, z), \\ 1 & \text { otherwise. }\end{cases}
$$

Then for any integers $p$ and $q$, there is a linked surface $F$ such that

$$
\Phi_{\theta}(F)=t^{p}+t^{-p}+t^{q}+t^{-q}+t^{p+q}+t^{-p-q}+21 .
$$

5.2. Topological application: noninvertibility. Surface braid theory was used in [4] and [5] to evaluate the invariants for some knotted surfaces. The theory is a generalization of braid theory in classical knot theory, and the fundamental theorems such as Alexander's, Artin's and Markov's theorems have been generalized to dimension 4 [20, 7]. Planar graphs called braid charts are used to obtain the following topological application of the invariant.

Theorem 5.7. For a certain cocycle in $Z^{3}\left(R_{3}, \mathbf{Z}_{3}\right)$, the 2-twist spun trefoil and its orientation reversed counterpart have the invariants $6+12 t$ and $6+12 t^{2}$ respectively. In particular, the cocycle invariant detects the noninvertibility of the 2-twist spun trefoil.

In [5], we also show how to compute the invariants using movie descriptions of knotted surfaces.

\section{REFERENCES}

1. Baez, J.C.; Langford, L., 2-tangles, Lett. Math. Phys. 43 (1998), no. 2, 187-197. CMP 98:08

2. Baez, J.; Langford, L., Higher-dimensional algebra IV: 2-tangles, to appear in Adv. Math, preprint available at http://xxx.lanl.gov/abs/math.QA/9811139

3. Brieskorn, E., Automorphic sets and singularities, Contemporary Math. 78 (1988), 45-115. MR 90a:32024

4. Carter, J.S.; Jelsovsky, D.; Kamada, S.; Langford, L.; Saito, M., Quandle cohomology and state-sum invariants of knotted curves and surfaces, preprint at http://xxx.lanl.gov/abs/math.GT/9903135

5. Carter, J.S.; Jelsovsky, D.; Kamada, S.; Saito, M., Computations of quandle cocycle invariants of knotted curves and surfaces, preprint at http://xxx.lanl.gov/abs/math.GT/9906115

6. Carter, J.S.; Kauffman, L.H.; Saito, M., Structures and diagrammatics of four dimensional topological lattice field theories, Advances in Math. 146 (1999), 39-100. CMP 2000:01

7. Carter, J.S.; Saito, M., Knotted surfaces and their diagrams, the American Mathematical Society, 1998. MR 98m:57027 
8. Carter, J.S.; Saito, M., On formulations and solutions of simplex equations, Internat. J. Modern Phys. A 11 (1996), no. 24, 4453-4463. MR 98g:17016

9. Carter, J.S.; Saito, M., Canceling branch points on the projections of surfaces in 4-space, Proc. AMS 116, 1 (1992), 229-237. MR 93i:57029

10. Dijkgraaf, R., and Witten, E., Topological gauge theories and group cohomology, Comm. Math. Phys. 129 (1990), 393-429. MR 91g:81133

11. Fenn, R.; Rourke, C., Racks and links in codimension two, Journal of Knot Theory and Its Ramifications 1 (1992), no. 4, 343-406. MR 94e:57006

12. Fenn, R.; Rourke, C.; Sanderson, B., Trunks and classifying spaces, Appl. Categ. Structures 3 (1995), no. 4, 321-356. MR 96i:57023

13. Fenn, R.; Rourke, C.; Sanderson, B., James bundles and applications, preprint found at http://www.maths.warwick.ac.uk/ bjs/

14. Fox, R.H., A quick trip through knot theory, in Topology of 3-Manifolds, Ed. M.K. Fort Jr., Prentice-Hall, 1962, pp. 120-167. MR 25:3522

15. Jones, V.F.R., Hecke algebra representations of braid groups and link polynomials, Ann. of Math. 126 (1989), 335-388. MR 89c:46092

16. Joyce, D., A classifying invariant of knots, the knot quandle, J. Pure Appl. Alg., 23, 37-65. MR 83m:57007

17. Hartley, R., Identifying noninvertible knots, Topology 22 (1983), 137-145. MR 85c:57003

18. Hillman, J.A., Finite knot modules and the factorization of certain simple knots, Math. Ann. 257 (1981), no. 2, 261-274. MR 83c:57009

19. Kamada, S., Surfaces in $\mathbf{R}^{4}$ of braid index three are ribbon, Journal of Knot Theory and Its Ramifications 1 (1992), 137-160. MR 93h:57039

20. Kamada, S., A characterization of groups of closed orientable surfaces in 4-space, Topology 33 (1994), 113-122. MR 95a:57002

21. Kamada, S., 2-dimensional braids and chart descriptions, Topics in Knot Theory (Erzurum, 1992), 277-287, NATO Adv. Sci. Inst. Ser. C Math. Phys. Sci., 399, Kluwer Acad. Publ., Dordrecht, 1993. MR 95g:57022

22. Kauffman, L.H., Knots and physics, World Scientific, Series on knots and everything, vol. 1, 1991. MR 93b:57010

23. Kawauchi, A., A survey of knot theory, Birkhauser, 1996. MR 97k:57011

24. Kawauchi, A., The invertibility problem on amphicheiral excellent knots, Proc. Japan Acad., Ser. A, Math. Sci. 55 (1979), 399-402. MR 81b:57003

25. Matveev, S., Distributive groupoids in knot theory, Mat. Sb. (N.S.) 119(161) (1982), no. 1, 78-88, 160. (Russian) MR 84e:57008

26. Murasugi, K., Knot theory and its applications, Translated from the 1993 Japanese original by Bohdan Kurpita. Birkhauser Boston, Inc., Boston, MA, 1996. MR 97g:57011

27. Neuchl, M., Representation Theory of Hopf Categories, to appear in Adv. in Math. under the title Higher-dimensional algebra VI: Hopf categories. Available at: http://www.mathematik.uni-muenchen.de/ neuchl

28. Ruberman, D., Doubly slice knots and the Casson-Gordon invariants, Trans. Amer. Math. Soc. 279 (1983), no. 2, 569-588. MR 85e:57025

29. Trotter, H.F., Noninvertible knots exist, Topology 2 (1964), 341-358. MR 28:1618

30. Roseman, D., Reidemeister-type moves for surfaces in four dimensional space, Knot Theory, Banach Center Publications 42 (1998), 347-380. MR 99f:57029

31. Rosicki, Witold, Some simple invariants of the position of a surface in $\mathbf{R}^{4}$, Bull. Polish Acad. Sci. Math. 46(4) (1998), 335-344. MR 99h:57050

32. Rudolph, L., Braided surfaces and Seifert ribbons for closed braids, Comment. Math. Helv. 58 (1983), no. 1, 1-37. MR 84j:57006

33. Rolfsen, D., Knots and links, Publish or Perish Press (Berkley 1976).

34. Wakui, M., On Dijkgraaf-Witten invariant for 3-manifolds, Osaka J. Math. 29 (1992), 675696. MR 95e:57033

35. Zeeman, E.C., Twisting spun knots, Trans. A.M.S. 115 (1965), 471-495. MR 33:3290 
Department of Mathematics, University of South Alabama, Mobile, AL 36688

E-mail address: carter@mathstat.usouthal.edu

Department of Mathematics, University of South Florida, Tampa, FL 33620

E-mail address: jelsovsk@math.usf.edu

Department of Mathematics, Osaka City University, Osaka 558-8585, JAPAN

Current address: Department of Mathematics, University of South Alabama, Mobile, AL 36688

E-mail address: kamada@sci.osaka-cu.ac.jp

E-mail address: skamada@mathstat.usouthal.edu

Department of Mathematics, University of Wisconsin at River Falls, River Falls, WI 54022

E-mail address: laurel.langford@uwrf.edu

Department of Mathematics, University of South Florida, Tampa, FL 33620

E-mail address: saito@math.usf.edu 